Recepción: 15/01/2019

Aceptación: 19/03/2019

Publicación: 05/06/2019

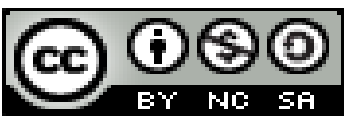

Ciencias económicas y empresariales

Artículos de investigación

\title{
Modelo de desarrollo emergente para la economía popular solidaria
}

\section{Emerging development model for the popular solidarity economy}

\section{Modelo de desenvolvimento emergente para a economia solidária popular}

Jorge Oswaldo Quevedo-Vázquez I
joquevedov@ucacue.edu.ec

Rolando Patrício Andrade-Amoroso II

randradea@ucacue.edu.ec

Tomas Eleazar Camacho-Calero ${ }^{\text {III }}$ tecamachoc60@est.ucacue.edu.ec

\author{
Patricia Guadalupe Guamán-Herrera IV \\ pggherrera.04@hotmail.com
}

Karen Denisse Manzanares-Ordoñez V
karenman96.km@gmail.com

Correspondencia: joquevedov@ucacue.edu.ec

\footnotetext{
${ }^{\text {I }}$ Magíster en Administración de Negocios, Diploma Superior en Práctica Docente Universitaria, Licenciado en Economía y Finanzas, Economista, Subdirección de Posgrados, Universidad Católica de Cuenca. Cuenca, Ecuador.

${ }^{\text {II }}$ Magíster en Administración de Empresas Mención en Recursos Humanos y Marketing, Auditor, Contador Público, Ingeniero Empresarial, Docente de la Universidad Católica de Cuenca. Unidad Académica de Administración Facultad de Contabilidad y Auditoría. Cuenca, Ecuador.

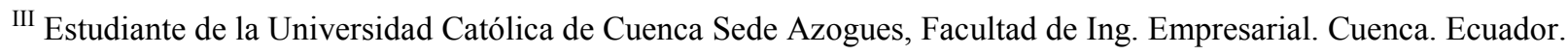

IV Estudiante de la Universidad Católica de Cuenca Sede Azogues, Facultad de Ing. Empresarial. Cuenca. Ecuador.

${ }^{\vee}$ Estudiante de la Universidad Católica de Cuenca Sede Azogues, Facultad de Ing. Empresarial. Cuenca. Ecuador.
} 


\title{
Resumen
}

El propósito de la investigación fue comprender las tácticas estratégicas de gestión desde la autonomía y empoderamiento popular y solidario, emergido desde la nueva significación de los sujetos involucrados, mediante la aplicación de un proceso hermenéutico. Los fundamentos teóricos están sustentados en Nyssens (2005) en lo que respecta a economía popular y la LOEPS (2011), referente a economía solidaria. Se desarrolló bajo el paradigma cualitativo de Flores y Tabón (2006), con un diseño emergente expuesto por Payares (2008) y Morse (1994). El método se ejecutó bajo los dos niveles descriptivos del enfoque fenomenológico empírico. Van Kaam (1966); Giorgi (1985). La técnica para recabar la información fue a través de la entrevista semiestructurada mediante un guion, Palella y Martins (2006). Los informantes Clave son 4 sujetos propietarios de empresas que cohabitan en distintas zonas populares de la ciudad de Manta, Ecuador- Sandín (2010). La técnica de análisis fue la hermenéutica Martínez (1996). Dentro de los hallazgos, los investigadores han creado un modelo para trascender el desarrollo emergente de la economía popular y solidaria desde la autonomía y empoderamiento popular; el mismo está compuesto por 4 ejes denominados: Sensibilización, Formación, Acción y Seguimiento, emergido de la resignificación de los versionantes al expresar la necesidad de profundizar los aspectos inherentes al abordaje que se le da al proceso económico comunitario. Entre las reflexiones finales destaca el compromiso real de los pobladores por dirigir procesos autogestionarios, inmersos en el respeto, la justicia, la paz, la equidad y ética e impulsando el trabajo voluntario bajo la concepción de una cultura organizativa con un nuevo esquema de relaciones de intercambio.

Palabras Claves: Desarrollo emergente; economía popular solidaria.

\begin{abstract}
The purpose of the research was to understand the strategic tactics of management from the autonomy and popular empowerment and solidarity, emerged from the new meaning of the subjects involved, through the application of a hermeneutical process. The theoretical foundations are supported by Nyssens (2005) in regard to popular economy and the LOEPS (2011), referring to solidarity economy. It was developed under the qualitative paradigm of Flores y Tabón (2006), with an emergent design presented by Payares (2008) and Morse (1994). The method was
\end{abstract}


executed under the two descriptive levels of the empirical phenomenological approach. Van Kaam (1966); Giorgi (1985). The technique for gathering the information was through the semistructured interview through a script, Palella and Martins (2006). Key informants are 4 owners of companies that cohabit in different popular areas of the city of Manta, Ecuador-Sandín (2010). The technique of analysis was the hermeneutics Martínez (1996). Within the findings, researchers have created a model to transcend the emerging development of the popular and solidarity economy from the autonomy and popular empowerment; it is composed of 4 axes called: Sensitization, Training, Action and Follow-up, emerged from the resignification of the versionantes when expressing the need to deepen the inherent aspects to the approach that is given to the community economic process. Among the final reflections highlights the real commitment of villagers to lead self-managed processes, immersed in respect, justice, peace, equity and ethics and promoting voluntary work under the conception of an organizational culture with a new relationship scheme of exchange

Keywords: Emerging development; popular solidarity economy.

\section{Resumo}

O objetivo da pesquisa foi compreender as táticas de gestão estratégica de autonomia e empoderamento popular e solidária, surgiu a partir do novo significado dos sujeitos envolvidos, através da aplicação de um processo hermenêutico. Os fundamentos teóricos são suportados por Nyssens (2005) no que diz respeito à economia popular e LOEPS (2011) a respeito da economia solidária. Ele foi desenvolvido sob o paradigma qualitativo e tabon Flores (2006), com um design emergente exposto por Payares (2008) e Morse (1994). O método foi executado sob os dois níveis descritivos da abordagem fenomenológica empírica. Van Kaam (1966); Giorgi (1985). A técnica de coleta de informações foi através de entrevistas semi-estruturadas com um hífen, Palella e Martins (2006). Informantes-chave são 4 proprietários assuntos de negócios que coabitam em diferentes áreas populares da cidade de Manta, Equador Sandin (2010). A técnica de análise foi a hermenêutica Martínez (1996). Entre as conclusões, os pesquisadores criaram um modelo de transcender o desenvolvimento emergente de economia popular e solidária de autonomia e empoderamento populares; ele é composto de 4 eixos chamados: Conscientização, Educação, Ação e Monitoramento, surgiu a partir da redefinição do versionantes para expressar a 
necessidade de aprofundar os aspectos inerentes à abordagem que é dada ao processo comunitário. Entre os pensamentos finais destaca o compromisso real das pessoas para direcionar processos imersos autogestionadas no respeito, justiça, paz, equidade e ética e promover o trabalho voluntário sob o conceito de uma cultura organizacional com um novo quadro de relações Troca

Palavras-chave: Desenvolvimento emergente; economia solidária popular.

\section{Introducción}

En el mundo globalizado de hoy, se ha impuesto una sociedad capitalista, conformando una economía y una sociedad "financiarizada", "virtualizada" y "desmaterializada" (Schujman, 2004), en el que se cercena lo pactado en la asunción universal establecido en la Carta internacional de Derechos Humanos, con pensamiento neoliberal y feroz en el tratamiento del mercado y la distribución y comercialización de los productos, lo cual repercute directamente en el consumo y la creación de empleos.

En tales circunstancias, se origina la organización de un sector cuya premisa es cubrir inicialmente las necesidades alimentarias y económicas sin adscribirse a los entes públicos o privados en América Latina con la presencia de La Unión de Naciones Suramericanas (UNASUR) y La Comunidad de Estados Latinoamericanos y Caribeños (CELAC), a partir de la praxis de sus pueblos como un espacio alternativo de recuperar empresas, redes de comercialización de la economía doméstica, agricultura familiar rural y urbana, y muchas otras manifestaciones económicas de solidaridad, reciprocidad y don que se renuevan y se modifican permanentemente entendida dentro de un concepto polisémicos de "Economía popular", "Economía del Trabajo", "Economía Solidaria” y "Economía Comunitaria” (Chaves, Monzón et al., 2012: 23).

A partir del 2008, se generaron cambios políticos en Ecuador que consistieron inicialmente en la aprobación de la nueva constitución de Montecristi que es la carta Magna por la que se rige el país, la cual trajo consigo la estatización del "sistema económico social y solidario" con el "reconocimiento al ser humano como sujeto y fin", ampliamente dispuesto en su artículo 283 al propender "una relación dinámica y equilibrada entre sociedad, Estado y mercado, en armonía 
con la naturaleza; y tiene por objetivo garantizar la producción y reproducción de las condiciones materiales e inmateriales que posibiliten el buen vivir".

Adicionalmente, se crean instituciones como la Superintendencia de Economía Popular y Solidaria (SEPS) y el Instituto Nacional de Economía Popular y Solidaria (IEPS), junto a leyes, normas y reglamentos como la Ley Orgánica de la Economía Popular y Solidaria y del Sector Financiero Popular y Solidario (LOEPS) y el Reglamento General de la Ley Orgánica de la Economía Popular y Solidaria y del Sector Financiero Popular y Solidario que contextualizan jurídicamente la puesta en práctica de la economía asumida en la nación para la "distribución equitativa, respeto a la entidad cultural, autogestión, equidad de género, comercio justo, responsabilidad social y ambiental, búsqueda del bien común y la prelación del trabajo sobre el capital", tal y como se dispone en la LOEPS (2011: 3).

A pesar de esta legislación y los esfuerzos del estado, son muy pocas las empresas de la ciudad de manta, Ecuador que se dedican a explotar la economía popular y solidaria de manera correcta y profunda; por eso, a juicio de los investigadores es a todas luces urgente y necesario un modelo sencillo de fácil lectura y aplicación para los interesados que permita comprender las tácticas estratégicas de gestión desde la autonomía y empoderamiento popular y solidario, emergido desde la nueva significación de los sujetos involucrados.

Bajo esa perspectiva, se estudia la categoría Economía Popular Solidarias con el objetivo de realizar el contraste de teórico durante el proceso interpretativo. Para Robbins (1932), la economía es "la ciencia que estudia la conducta humana como una relación entre fines y medios escasos que tienen usos alternativos". Este vínculo permite al individuo realizar actividades que les acerque a la obtención de bienes y beneficios con herramientas que se adecuan para ello.

Entonces, la Economía popular es el conjunto de actividades económicas organizadas por los pobladores según Nyssens (2005:9). Es decir, un despliegue de personas miembros de una localidad específica que se integran para participar en el desarrollo económico, endógeno, inclusivo, autogestionario y sustentable de un espacio geográfico dado que pertenece al pueblo con arraigo en su identidad, en asociación y colaboración colectiva como actores sociales en resistencia al sistema hegemónico para avanzar hacia una economía plural. 
Por su parte, en el siglo XIX la economía solidaria surge a partir de las organizaciones de forma cooperativa, asociativa y de ahorro y crédito, para dar respuesta a las necesidades económicas de la población (Guridi et al, 2014:15), en el que se incorpora el "conjunto de emprendimientos de producción, transformación, comercialización y servicios de grupos de personas (Arcos et al, 2011: 9), con la firme intención de afianzar el crecimiento territorial y mejorar la calidad de vida de sus habitantes.

En este orden la LOEPS (2011), define la economía solidaria como:

La forma de organización económica, donde sus integrantes, individual o colectivamente, organizan y desarrollan procesos de producción, intercambio, comercialización, financiamiento y consumo de bienes y servicios, para satisfacer necesidades y generar ingresos, basadas en relaciones de solidaridad, cooperación y reciprocidad, privilegiando al trabajo y al ser humano como sujeto y fin de su actividad, orientada al buen vivir, en armonía con la naturaleza, por sobre la apropiación, el lucro y la acumulación de capital.

En palabras de (De Melo 2004:397), la solidaridad que tenemos que desarrollar es una solidaridad decolonizadora, "interclasista hacia el otro (pobre, o racial e ideológicamente diferente), incluso hacia las demás formas de vida (la naturaleza)"... para afianzar una “economía transformadora que reivindica la praxis alternativa que se construye partir de la "economía popular" de las "unidades domésticas", del ámbito local y regional, para transitar por las actividades asociadas que se expresan en la economía solidaria, para poder construir "otra economía" y alejarse de una vez por todas del colonialismo eurocéntrico.

\section{Materiales y Métodos}

En el presente estudio se empleó el paradigma cualitativo, en el cual el pensamiento y la realidad están intrínsecamente ligados dado que se tiene la convicción sobre una situación modelada y construida por la razón de acuerdo a un fenómeno en el cual se le imprime una perspectiva propia que abre la posibilidad para conocerla. En ese sentido, Flores y Tabón (2006), la definen como un proceso de valoración que parte de la fuerza cognitiva del indagador, que simultáneamente se expresa en un lenguaje intersubjetivo que desata sendas de discusión, persuasión y consenso; 
siempre y cuando los nuevos aspectos comprendidos y mostrados revelen un nuevo orden y una nueva significación para los interlocutores.

A su vez, está configurado con un diseño emergente construido a la medida en que se avanzó la investigación en atención a lo expuesto por Payares (2008), cuando afirma que el problema inicial de la investigación, va reformulando constantemente una confirmación sobre los datos recogidos, contribuyendo a la interpretación del fenómeno y cuando se encuentran en desajustes importantes.

Asimismo, se trabajó con el método la fenomenológico, el cual enfatiza la vuelta a la reflexión y a la intuición para describir y clasificar las experiencias, tal como, es vivida, y se configura como conciencia (Morse, 1994). De allí, que este enfoque permitió describir y comparar lo teórico y práctico de la economía popular solidaria, ya que permite acceder a la descripción de esos escenarios desde la perspectiva de los que han sentido la vivencia cuyo valor y significación pone de manifiesto la solución de los problemas planteados.

El enfoque fenomenológico de la presente investigación es empírico porque involucra un regreso a la experiencia para obtener descripciones comprensivas que proporcionan las bases para un análisis estructural reflexivo que retrata las esencias de la práctica. "Busca descubrir y dilucidar los fenómenos de comportamiento como ellos se manifiestan por si mismos en su percepción inmediata" (Van Kaam, 1966, p.15). En ese orden de ideas, la perspectiva fenomenológica tiene una especial relevancia epistemológica, al valorar los nexos de interdependencia que tienen los elementos constituyentes de una entidad y los nexos de diferentes realidades, considerando además la interpretación como relativa y provisional; por tanto, la realidad necesita ser enfocada desde muchos ángulos diferentes para poder ser ilustrada y dilucidada adecuadamente.

Atendiendo a las consideraciones anteriores, Giorgi (1985:69), señala los dos niveles descriptivos del enfoque fenomenológico empírico: Nivel I: los datos originales están comprendidos por simples descripciones obtenidas a través de preguntas y diálogos abiertos y cerrados. Nivel II: el investigador describe las estructuras de la experiencia basados en el análisis reflexivo e interpretación de la investigación de los relatos o historia de los participantes. En los términos de 
Giorgi, primero se narran las voces de los informantes y luego se caracterizan esas expresiones desde la praxis del investigador.

La técnica utilizada para recabar la información, fue la entrevista semiestructurada a través de guion como instrumento de indagación. Según Palella y Martins (2006:123) "es una técnica destinada a obtener información confiable de varias personas, a través de un diálogo". Esta conversación contribuye a la aportación de datos de interés que enriquecen el estudio. Los informantes Clave son 4 sujetos que son propietarios de empresas que cohabitan en distintas zonas populares de la ciudad de Manta, Ecuador, por ser entes que están relacionados directamente con el área de conocimiento o especialidad de la categoría a evaluar. Sandín (2010). Es decir, son los que generan el mayor cumulo de información valiosa, porque permiten evaluar de manera cualitativa los contextos prácticos del estudio a través de las interacciones.

La técnica de análisis es la hermenéutica mediante el "descubrimiento de los significados de las cosas" Martínez (1996), pues, a través de los testimonios recibidos de los informantes como sujeto del objeto a investigar en una directa interacción los autores pidieron realizar un ejercicio exegético que junto a la praxis del tema les permitió la introspección, interpretación y comprensión del tópico en estudio en el que finalmente subyace la creación de un modelo emergente para la economía popular solidaria.

\section{Hallazgos}

Las apreciaciones de los entrevistados muestran una clara imagen que sobre la gestión de la economía solidaria posen los informantes. Ellos manifiestan que algunas personas se involucran con las comunidades para sus intereses particulares o lo que es lo mismo, como trampolín para hacer carrera política y hacerse popular, hecho que genera desconfianza. Expresan además, que no se resisten a los cambios si eso conlleva a la mejoría económica pero a los ojos de los investigadores, es notoria la desmotivación que remite la merma de sus ámbitos de acción en cualquier intento por mejorar los procesos y funciones específicas para incrementar la participación ciudadana como parte de la organización social

Por ello, urge implementar un modelo de desarrollo para la economía popular y solidaria emergido de la resignificación de los versionantes al expresar la necesidad de profundizar los aspectos inherentes al abordaje que se le da al proceso económico comunitario en aras de 
fortalecer mecanismos de relación entre ellos mismos y los entes externos, con el cual se aspira elevar la potencialidad de acciones para resolver situaciones complejas en el colectivo.

En ese sentido, los investigadores han creado un modelo que se presenta a continuación para trascender a las tácticas estratégicas de gestión desde la autonomía y empoderamiento popular; el mismo está compuesto por 4 ejes denominaos: Sensibilización, Formación, Acción y Seguimiento. Asimismo dos niveles, de Arranque y Operativo, en ese orden que finalmente se subyugan al alcance de los objetivos, partiendo de sus dos pilares que son Economía Popular y Economía Solidaria de manera transversal. (Ver Imagen 1).

\section{Objetivo General}

Fomentar intercambios sociales con sentido crítico y participativo de la ciudadanía en materia de política constructiva, popular y solidaria con miras a la transformación democrática a través del dialogo y la cooperación de los miembros de la comunidad.

\section{Objetivos Específicos}

- Fortalecer el sentido de lo público.

- Contribuir al desarrollo de los principios de transparencia, responsabilidad, eficacia, eficiencia e imparcialidad y participación ciudadana en la toma de decisiones.

- Constituir un espacio de interlocución entre la ciudadanía.

- Servir como insumo para ajustar proyectos y planes de acción de manera que responda a las necesidades y demandas de la comunidad.

- Integrar los aportes y propuestas de la ciudadanía, una vez conocidas, validadas y concertadas con la interacción interinstitucional.

- Facilitar a la ciudadanía la participación en la planeación y control del desarrollo de los planes, proyectos y programas comunitarios e institucionales.

\section{Metas}

- Identificar el perfil económico del territorio.

- Fomentar la participación e información de la ciudadana en los asuntos locales.

- Crear un mecanismo para la de promoción del desarrollo económico endógeno y local.

\section{4}

Pol. Con. (Edición núm. 34) Vol. 4, No 6, junio 2019, pp. 26-37, ISSN: 2550 - 682X 
- Administrar efectivamente los recursos propios.

- Establecer contactos con niveles del gobierno.

- Promover la creación de pequeñas empresas.

- Aumentar la productividad comunitaria.

Imagen 1. Modelo de Desarrollo emergente para la economía Popular Solidaria

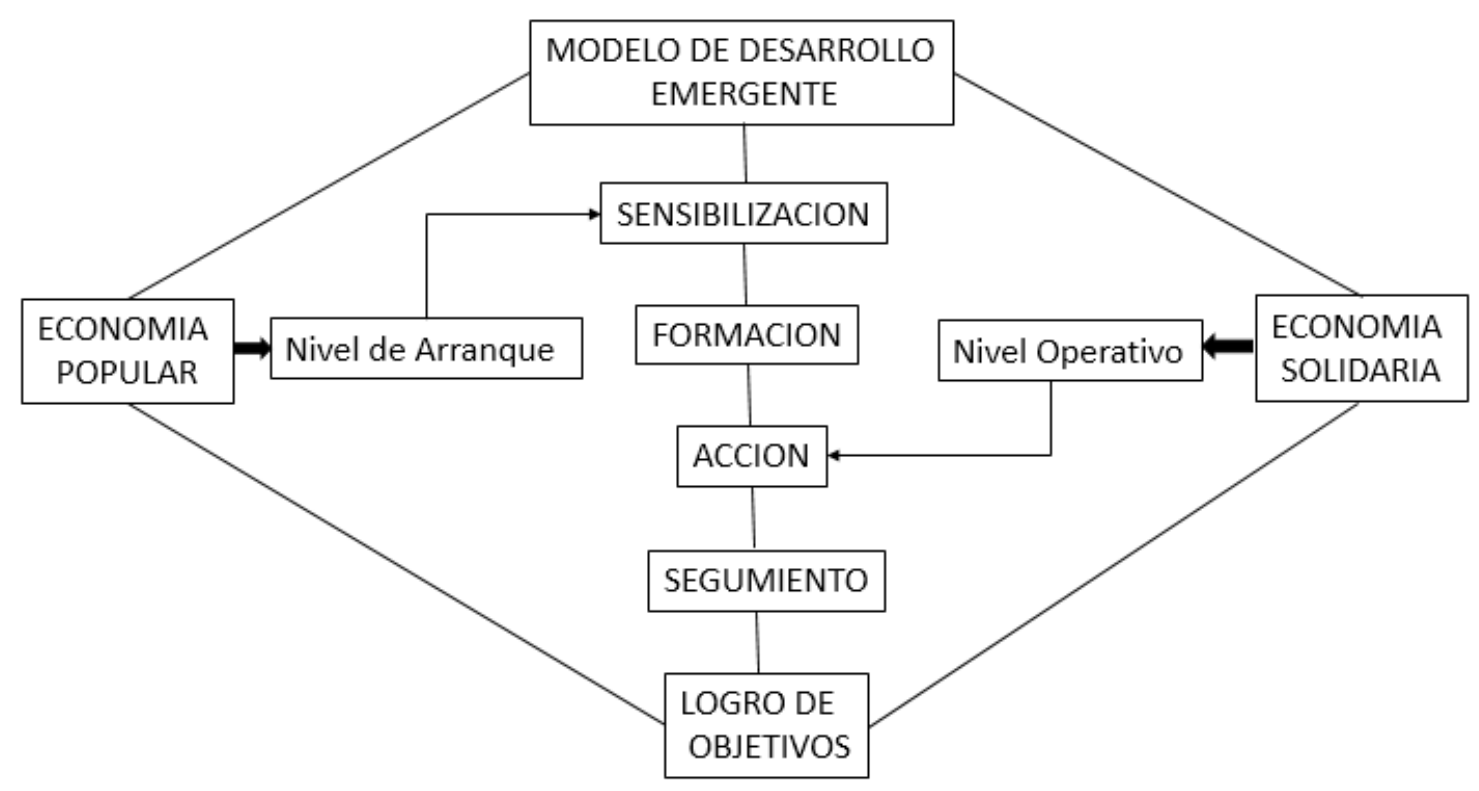

Fuente: Autores (2019). Elaboración propia.

\section{Reflexión Final}

Dada la capacidad de redimensión de la gestión que el modelo sugiere se propone tácitamente la resolución de problemas y/o necesidades en situaciones problematizadas de la comunidad, para generar impacto en la colectividad, de tal forma que las actividades que se despliegan desde este ámbito estén orientadas principalmente a integrar acciones desde diferentes perspectivas para enfrentar multidisciplinariamente e intersectorialmente los problemas y las situaciones que aún permanecen sin resolver a nivel de la comunidad.

A pesar de que aún persiste la existencia de muchas y muy variadas situaciones de desapego y falta de empatía, este modelo puede iniciar un compromiso real de los pobladores por dirigir procesos autogestionarios, inmersos en el respeto, la justicia, la paz, la equidad y ética e impulsando el trabajo voluntario bajo la concepción de una cultura organizativa con un nuevo 
esquema de relaciones de intercambio ganar- ganar y alcanzar un máximo de organización y disciplina de control y seguimiento efectivo de la economía sustentable con la aplicación inmediata y sostenida del mismo.

\section{Referencias Bibliográficas}

Alburquerque, F. (2004). Elementos para repensar el concepto de autogestión Albuquerque,

Chaves, R. (2007). La economía social como enfoque metodológico, como objeto de estudio y como disciplina científica". En Vuotto, M. (comp) Economía Social. Precisiones conceptuales y algunas experiencias históricas. Ed. Altamira. Buenos Aires.

Constitución de la republica de Ecuador (2008).

De Melo Lisboa, A. (2004). "Mercado Solidario". En "La otra economía”. Cattani, A. (Organizador). Ed. UNGS, Fundación OSDE y Altamira. Buenos Aires.

Flores O., Tabón. (2006). Evaluación pedagógica y cognición. Colombia. McGRAW-HILL Interamericana S.A.

Giorgi, A. (1985). Fenomenología y psicológica. Pittsburgh. Duchisne University Press.1985

Guridi, L. y Mendiguren, J. (2014). La Economía Social y Solidaria, y el Desarrollo Humano local $2^{\text {a }}$ edición Transformando los territorios desde la economía solidaria. Herramientas para el impulso de políticas públicas locales proyecto de REAS Euskadi ISBN: 978-84945538-6-8

Ley Orgánica de la Economía Popular y Solidaria (2011).

Martínez, M. (1996). Comportamiento humano: nuevos métodos de investigación. $2^{\mathrm{a}}$ edic. México: Trillas

Morse, J. (1994) El diseño de la Investigación Cualitativa. Editorial. Oaks, California: SAGE

Nyssens, M. (1997). El germen de una economía solidaria: Otra visión de la economía popular. El caso de Santiago de Chile. CIRIEC Espana: revista de economia pública, social y cooperativa, $(25), 63$. 
Payares, L. (2008). Plan de capacitación docente basado en estrategias de enseñanza y toma de decisiones para la unidad curricular física general para el IUTAG.

Robbins, L. (1944). Ensayo sobre la naturaleza y significación de la ciencia económica, Fondo de Cultura Económica, México.

Sandín (2010), Investigación Cualitativa en Educación; Fundamentos y Tradiciones. Editorial Mc Graw Hill. Interamericana de España S.A

Schujman, M. S. y Comps. Economía social y solidaria: praxis, vivencias e intenciones / Mario S. Schujman; Paulo Peixoto de Albuquerque; Kelly C. Pereyra, Karina Tomatis. - $1^{\text {a }}$ ed. Rosario. Ediciones Del Revés, (2014) 464 p. ISBN 978-987-29098-5-7

Van Kaam, A. L. (1966). Phenomenal analysis exemplified by a study of the experience of 'really feeling understood'. Journal of Individual Psychology, v.15, n.1, p.66-72. 